\title{
Measurement System for the Characterization of Magnetic Actuators on the base of Magnetic Stray Fields
}

\author{
Prof. Dr. Ing. Jörg Franke, M. Eng. Matthäus Brela, Ursula Pellkofer \\ Institute for Factory Automation and Production Systems \\ Friedrich-Alexander-University of Erlangen-Nuremberg, \\ Egerlandstraße 7-9, 91058 Erlangen, Germany \\ e-Mail: matthaeus.brela@faps.uni-erlangen.de
}

\begin{abstract}
Common defects in electromagnetic actuators due to the manufacturing process are heterogeneous magnetic properties of the materials, cracks and defects in the magnetic materials and parasitic air gaps in and directly around the magnetic circuit. Considering the high cost pressure, test systems must be adapted to the increasing complexity of the components and be able to detect defects completly in-situ. For these requirements the novel and non-destructive measurement system is suitable to monitor and assure quality of magnetic actuators within the manufacturing process. The measuring principle is based on the characterization of the magnetic circuit according to the magnetic stray fields that can be quantified by hall-sensor measurement outside the circuit. The intensity of that stray field is dependent on the magnetic flux density in the magnetic circuit. Therefore parameter variations, for example due to the components assembling can be detected in an early stage of the manufacturing process automatically and "in-line".
\end{abstract}

Key words: Magnetic Stray Field, Measurement System, Electromagnetic Actuators, Non-Destructive Measurement, Manufacturing

\section{Motivation}

An electromagnetic actuator has to reach a specific magnetic force at a given coil current. Because of the reluctance principle electromagnets have a characteristic mode of action which inducts an anchor movement in only one direction (active direction of movement) with the help of the magnetic field forces. However, the reset of the anchor (passive direction of movement), must be exerted by external forces (for example spring forces). Together with other factors the magnetic resistance of the entire magnetic circuit has to be ensured within defined tolerance or kept as low as possible. Parasitic air gaps in the magnetic circuit or exceeded tolerances of the magnetic material properties have a negative effect on the reluctance of the whole actuator and therefore the useful magnetic flux. [1]

The aim of the research was to develop a measurement system for investigation and evaluation of the magnetic properties of an electromagnetic actuator to ensure the quality by an in-situ testing during the manufacturing process. The restriction was to avoid implementing sensors in the magnetic circuit, because this would affect the reluctance of the magnetic circuit itself. In addition, special attention was paid to develop an automated, rapid and non-destructive measurement method.

\section{State of the Art}

Currently, electromagnetic actuators are usually tested in the context of a final exam to measure their functionality. However, not the forcegenerating magnetic field strength is measured. Normally the resulting force is being tested for example the pressure of a hydraulic media or the force profile. The test ensures that the electromagnetic actuator is within the predetermined tolerance. Usually it cannot be determined which component or assemblysteps are the cause of the error. In that way the possibility to react on defects in an early stage of manufacturing can be lost.

Up to now the measurement of magnetic flux found no application in the characterization of electromagnetic actuators [1]. There were approaches (by the Steinbeis Transfer Center in IImenau [3]) to determine and to monitor the hysteresis through the implementation of Hallelements in the magnetic circuit. However, these had the disadvantage of increased production costs due to additional 
manufacturing steps. On the other hand an additional parasitic air gap within the magnetic circuit was required that increases the magnetic resistance. [2] This problem was solved by the developed measurement system using the magnetic stray field outside the magnetic circuit to characterize the electromagnetic actuators.

\section{Construction of Electromagnetic Actuators}

Electromagnets and electromagnetic drive units are available in many different designs which can be traced back to a few basic types. These are the cylindrical magnet, the U-shape-magnet or the E-shape-magnet. Iron circle (core material), the excitation coil and the working air gap are the relevant elements for the functionality of each electromagnet. The magnetic circuit consists of an anchor as the movable part, a magnetic core (stator), which is connected fixed to the frame of the excitation coil and the working air gap (see Fig 1), in which the magnetic force is generated. Depending on the structural design of these functional elements, electromagnetic actuators can be designed with different magnetic forcestroke characteristics. They are dependent on the technical requirements of the electric drives. [1]

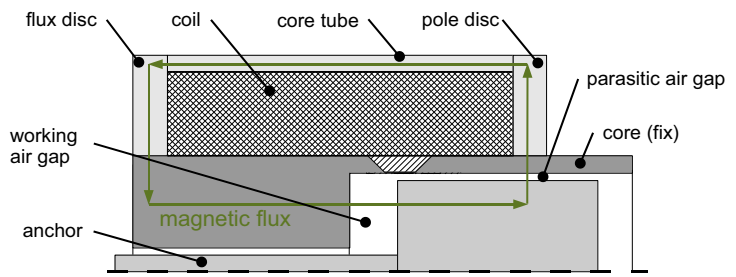

Fig. 1. Schematic drawing of a pot magnet showing its basic components and pointing out the difference between working and parasitic air gap.

\section{Manufacturing of Electromagnetic Actuators}

The components of the electromagnetic actuator are manufactured and assembled in a complex process to close the magnetic circuit. To keep the magnetic stray fields as small as possible and to reach the required force-strokecharacteristic, it is important to reduce the size of parasitic air gaps on the joining. For that reason a lot of know-how is required to realize the assembly process. Common defects in manufacturing of electromagnetic actuators are different magnetic properties of the materials, cracks and defects in the materials and parasitic air gaps in and directly around the magnetic circuit. Since the kind of potential failures and defects can be very different it is quite difficult to take all of them in the early development into account. Many types of errors occur only in the sample phase or after the start of production. After that date the error costs increase over-proportionately with the time that elapses until their discovery.

The aim of the research was the detection of different defect types based on the measurement of magnetic stray fields outside the magnetic circuit without damaging the electromagnetic valve. In this research the effect of the 3 main types were discovered:

- Differing magnetic properties of the fluxconducting materials in the magnetic circuit

- Air gaps or parasitic air gaps in the magnetic circuit

- Winding defects and micro cracks in the wire or in the insulation of the exciter coil.

\section{Theory}

The magnetic resistance (reluctance) of the sections of a magnetic circuit is proportional dependent to the magnetic properties of the flux-guiding material. By simplifying the magnetic conductive sections into square elements and the assumption that the magnetic flux passes the material homogeneous, the reluctance can be calculated as following:

$R_{m}=\frac{l}{\mu A}$

In a homogeneous magnetic field $(B=$ constant), the magnetic resistance of a magnetic circuit section can be determined by the ratio of its length $\mathrm{I}$, by its cross-sectional area $A$ and the permeability $\mu$. All magnetic resistances of the magnetic circuit can be combined to a magnetic resistance $R_{m}$. The magnetic flux in an ideal homogeneous magnetic circuit is given by the following equation:

$\phi=\frac{\theta}{R_{m}}$

Whereby $\theta$ is the magnetomotive force generated by the coil. Different magnetic properties of the flux-guiding material, for example the permeability or the hysteresis are reflected directly back to the magnetic resistance. [1] According to equation (2) these properties have a direct effect on the magnetic flux. In that equation the linear correlation between the coil and the magnetic flux can be seen. The magnetomotive force can be calculated by the Number of windings $\mathrm{N}$ and the current I applied to the coil. 
$\theta=N I$

The greatest influences on the magnetic circuit have parasitic air gaps and the working air gaps. Air has a low permeability, and thus an air gap in the magnetic circuit is equal to a high reluctance. Even small manufacturing tolerances and thereby occurring parasitic air gaps lead to significant fluctuations in the total reluctance. There are also parasitic air gaps being functionally relevant in order to prevent the friction of the movable anchor from becoming unintentional high caused by tolerances in the joining process. All of them are an additional magnetic resistance and reducing the magnetic flux, and thus the useful flux in the working air gap (see Fig. 1).

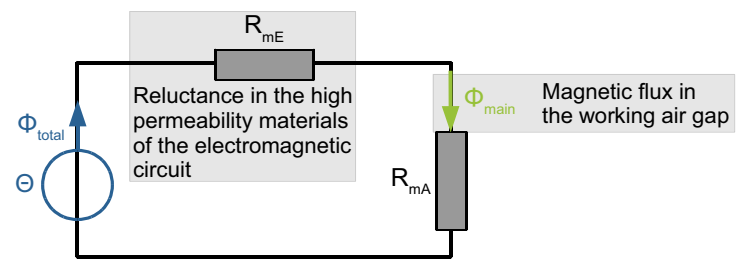

Fig. 2. Equivalent circuit of a simple magnetic circuit.

\section{Method of Solution}

Under ideal conditions (ideal view) there is no magnetic flux outside the magnetic circuit. This ideal consideration is only valid under the assumption that the permeability of the air can be neglected compared to the permeability of the flux-guiding material. Thus, there are no magnetic stray fields and therefore the magnetic flux in the working air gap is uniform. This is one of the reasons why a measurement method for the characterization of magnetic circuits based on magnetic stray fields has not been established yet.

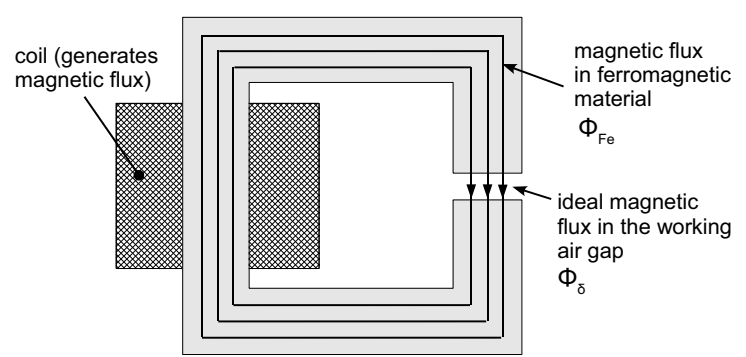

Fig. 3. Idealized figure of the magnetic flux in a simple electromagnetic circuit with a working air gap.

A more realistic view considers that stray magnetic fields in the working air gap cannot be neglected which means that the field distribution is assumed to be inhomogeneous. In that case the magnetic flux in the working air gap is composed of a parallel connection of the useful and stray magnetic flux.

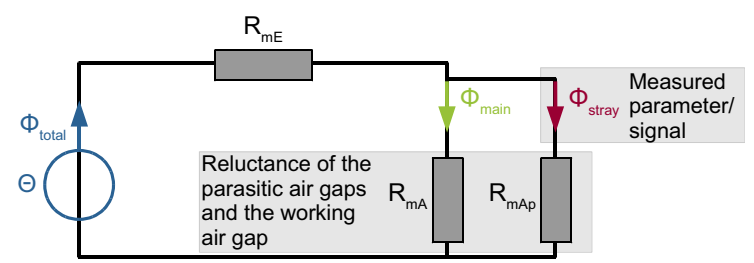

Fig. 4. Schematic equivalent circuit of a simple magnetic circuit, taking the magnetic flux leakage and the main flux into account.

$$
\phi=\frac{\theta}{\left(R_{m E}+R_{m A} * R_{m A p} /\left(R_{m A}+R_{m A p}\right)\right)}(4)
$$

$R_{m E}$ is the reluctance of the flux-carrying components of the magnetic circuit, $R_{m A p}$ the magnetic resistance of the magnetic stray fields and $R_{m A}$ of the magnetic air gap (useful flux). All effects of the production or factors affecting magnetic force in the magnetic circuit are reflected in the useful flux. Due to a correlation of magnetic stray fields see eg. (4) and see Figure 6 they are generally very suitable for an assessment of the useful magnetic flux. Additionally, the surface transitions are directly detectable by magnetic stray field. The solved problem was to detect the non-homogeneous stray fields and to be able to assign them to specific types of defect.

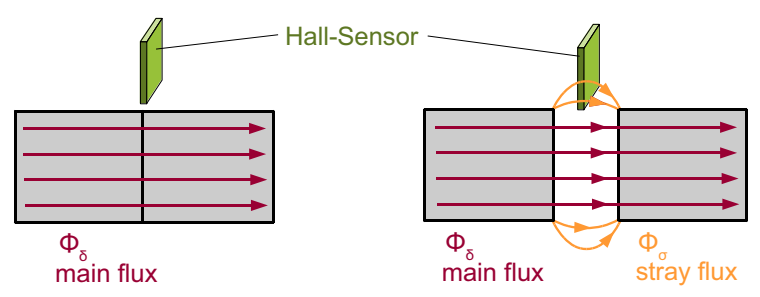

Fig. 5. Measureable stray fields using a HallSensor with and without an air gap between flux conducting parts.

\section{Sensor Technology}

To measure the magnetic fields, the Hall-Effect was used. The Gaussmeter was from Lakeshore Ltd. The Hall-Effect is named after its discoverer Edwin Hall. In a current-carrying conductor to which a stationary magnetic field is applied, along its cross-sectional area, a potential difference can be detected. The phenomenon is caused by the Lorentz-Force, which is exerted by a stationary magnetic field on moving charges. According to the right-hand rule, these charges are distracted. For this reason, at one side of the Hall element produces a surplus of electrons and at the other side an electron deficiency. This potential difference and the resultant voltage, which is 
named the Hall-Voltage, can be detected perpendicular to the current direction in the Hall element and perpendicular to the magnetic field.

The Hall voltage $U$ is dependent on the geometrical dimensions of the sensor element, a material constant, and the magnetic flux density, which acts perpendicular to the Hall sensor material. The only variable parameter is the magnetic flux density.

$U_{\text {Hall }}=\frac{A_{\text {Hall }} I B}{d}$

A is the Hall constant, which is a material constant of the Hall element, I represent the current, $B$ the magnetic flux density and $d$ is the geometric dimensions of the Hall element (see Fig. 6).

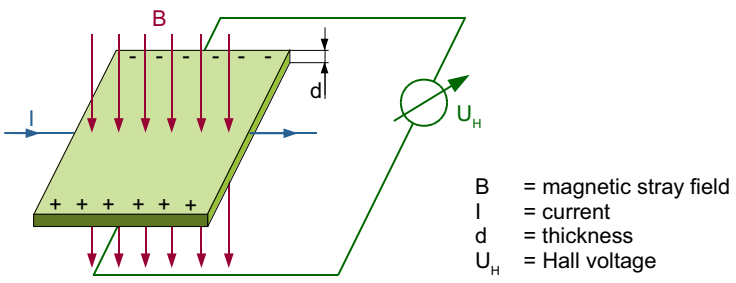

Fig. 6. Principle of a Hall-Sensor.

The measuring range of the Hall sensor is between 0.35 HTesla (350 mGauss) and 350 mTesla (350 kGauss). The resolution is $2 \%$ of the present range. The sensor has been selected on the basis of numerical simulation models that have been carried out with the software ANSYS FEMM Maxwell. The electromagnetic simulation allowed an estimation of the magnetic flux density or the magnetic stray fields in the surrounding area of the investigated actuators. The analysis showed that the magnetic stray field outside the magnetic circuit can be up to 10 mTesla.

\section{Measuring Device}

In the research project, a measurement system was developed, consisting of measurement unit, chuck controller, control unit and data processing.

The electromagnetic actuator will be placed in a holding block of the measuring device, and automatically tensioned. (see Figure 7, right). Over lateral stops, the valve is brought into a clearly defined position. The sensor, which is fixed in a clamping holder, will be moved by a double acting cylinder to the actuator. The whole unit together with the sensor clamping is the named sensor unit. By linear slides additional measuring positions can be approached. A stepper motor is responsible for the angular positioning.
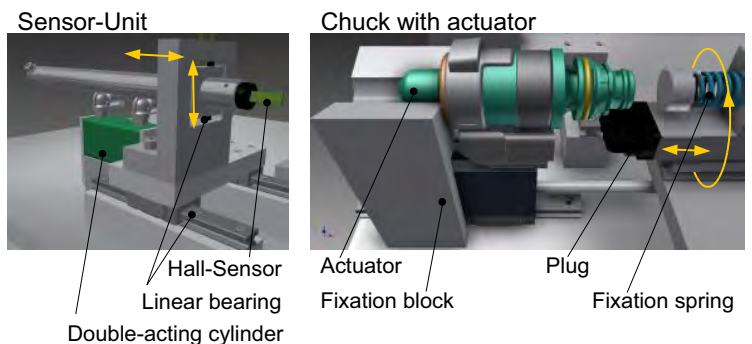

Fig. 8. CAD drawing of the Sensor Unit (left) and the clamping unit (right).

The actuator is contacted automatically. After the clamping step, a first energization is performed to negate the residual magnetism will be done and thus ensure consistent test conditions. The reason behind this procedure is the restoration of the magnetic characteristics in materials over time.

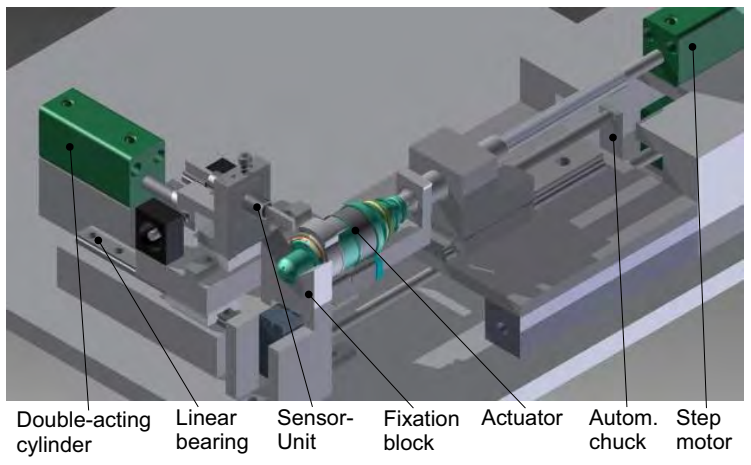

Fig. 8. CAD drawing of the Measuring device to detect stray magnetic fields on a pressure regulator.

In order to detect the hysteresis of the electromagnetic actuator a variable current or a constant current and the number of cycles can be defined before the measurement.

Fig. 9. Device to detect stray magnetic fields on a pressure regulator.

All components of the testing device are made of non-magnetic, diamagnetic or paramagnetic materials, predominantly aluminum. The reason is to avoid an influence of the surrounding material on the measurement result.

For the programming of the measuring position is an RS232 interface to the control unit available. Thus different measuring positions can be defined. The current profile can also be programmed via a LabView user-interface. The 
sensor data is read via an analog interface on the Teslameter and transferred to the LabView program. For good responding qualities the measurement and the sensor position data is written into a text file. After the measurement this data is converted in an Excel spreadsheet.

\section{Results of the Investigation}

To represent the measurement capability three cases are presented.

1) Simulation of a simplified model of an electromagnetic pressure regulator with ANSYS Maxwell. The goal is the theoretical proof of the measurement capability.

2) There were non-annealed test parts (anchor and magnet core) assembled in the magnetic circuit to influence the useful flux artificially.

3) In addition, investigations were carried out on electromagnetic pressure controllers which have not reached the required magnetic force for a given test current.

\section{Simulation of gaps in the magnetic circuit}

If gaps occur in the magnetic circuit then the local magnetic resistance is increased ( $\mathrm{RmAp}$ parasitic resistance, see fig. 10).

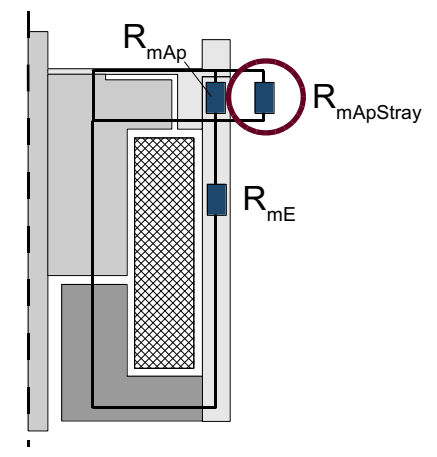

Fig. 10. Stray field measurements outside of the actuator with the variation of the test current. Difference in the hysteresis curve in annealed (gray) and non-annealed (blue) anchor.

This reduces the ratio of the reluctance in the magnetic circuit $R_{m A p}$ and the reluctance outside of the magnetic circuit $R_{\text {mApStray }}$. According to this prediction larger stray fields can be measured near the parasitic air gap. This is also confirmed by simulations (see fig. 11, left). Furthermore, it can be observed that the total magnetic flux decreases, which results on weaker external magnetic stray field (see fig. 11, right, upper red cloud).

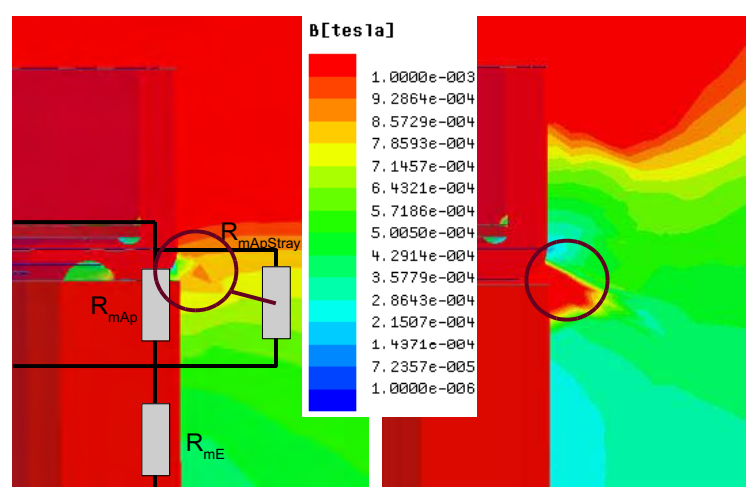

Fig. 11. Stray field simulations outside the actuator with the variation of the test current. Difference in the hysteresis curve in annealed (gray) and nonannealed (blue) anchor.

\section{Investigation of different material characteristics}

An increase of the coercive force results that the permeability is reduced in dependence on the magnetic flux. Due to this flatter hysteresis curve occur (see fig. 12 and eg. 1). From the hysteresis curve even more characteristic values are to be derived, which is currently being investigated in more detail.

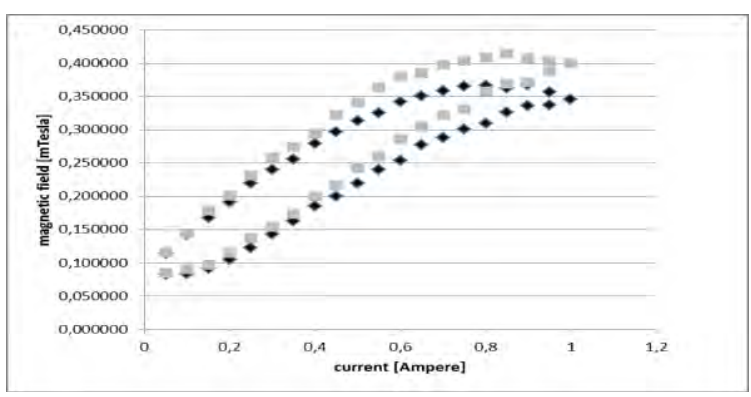

Fig. 12. Stray field measurements outside of the actuator with the variation of the test current. Difference in the hysteresis curve in annealed (gray) and non-annealed (blue) anchor.

This measuring method could find $100 \%$ of the non-annealed anchor or magnet cores. Thus early weaknesses in the production could be detected.

\section{Investigations on incorrectly produced parts}

Actuators that have not reached the necessary magnetic force during the test procedure are characterized by an increase in the total reluctance of the magnetic circuit. This has the consequence that the magnetic stray fields are also weakened. The exception is when the error occurs directly on the measuring position. In the case of fair gaps the strength of local stray field can also be increased.

In the investigation it was known that the defects originated from the damaged pole tube, 
thus they had no direct influence on the stray field at the measurement position. Therefore, the measurement results correlate with the useful flux in the working air gap. The measurements confirmed this theoretical approach. It can clearly be seen that actuators, which reached the magnetic force having higher magnetic stray field strength (see fig. 13).

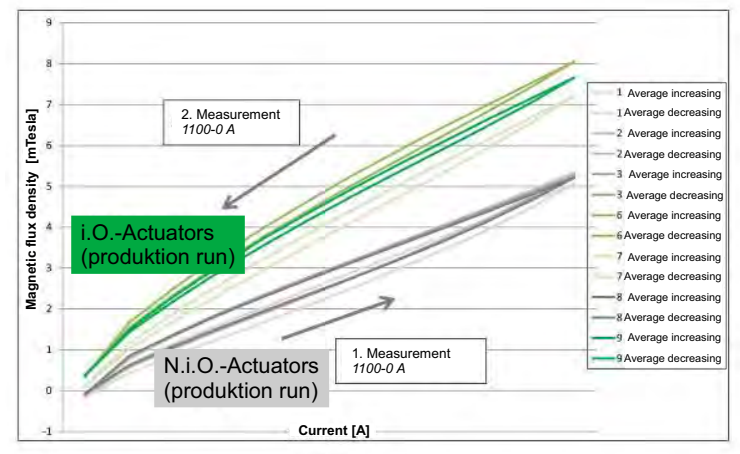

Fig. 13. Stray field measurements outside of the actuator with the variation of the test current. Difference in the hysteresis curve in annealed (gray) and non-annealed (blue) anchor.

All tested actuators that have not reached the magnetic force could be identified reliably at a specific current curve.

\section{Conclusion}

The study has shown that the developed measurement system or the innovative measuring method, based on the measurement of magnetic stray fields is suitable to characterize electromagnetic actuators. It has been shown theoretically and by practical experiments with the developed testing device that the stray fields correlate with certain defects. The outlook has to be to investigate more into the research of allocation and quantifying the different defect types. It makes sense to work closely together with the production and the development divisions of electromagnetic actuators to respond early on errors and their resulting images.

\section{References}

[1] Glet U.; Baumbach, J.: Ein neuartiges Verfahren zur Messung magnetischer Bauteile und Magnetaktoren. 30. IWK der TU IImenau, 2008

[2] Kallenbach E.: Elektromagnete, Grundlage, Berechnung, Entwurf und Anwendung, Wiesbaden, 2003

[3] Gadyuchko A.; Kallenbach E.: Magnetische Messung - Neue Wege der

Funktionsprüfung bei der Herstellung von Magnetaktoren. Beitrag zur VDI-Tagung - Klein- und Mikroantriebstechnik, Würzburg, 2010 